Artigo

\title{
Medidor de grandezas elétricas com acesso remoto
}

\author{
Bruno do Nascimento ${ }^{[1]}$ e Daniel Pablo Dantas Diógenes ${ }^{[2]}$ \\ ${ }^{[1]}$ UFERSA; brunonascimento777@gmail.com \\ ${ }^{[2]}$ UFERSA; Daniel.diogenes@ufersa.edu.br \\ Recebido: 08/07/2020; \\ Aceito: $09 / 08 / 2020$ \\ Publicado: 11/09/2020.
}

Resumo: O alto custo de energia elétrica devido a geração, distribuição e taxas e impostos traz um oneroso valor a renda de várias famílias, sendo necessário uma maior quantidade informações sobre o consumo de eletricidade de cada aparelho bem como de uma residência inteira. Atualmente existe no mercado aparelhos para medição, registro e análise de grandezas elétricas. O objetivo do presente trabalho é a criação de um protótipo para o registro e monitoramento de grandezas elétricas, destinado a implementação na área residencial. O projeto é baseado em um módulo PZEM-004T v3 com capacidade de até 100 A, usando um sensor de corrente não invasivo e uma placa de desenvolvimento ESPDUINO32 para recuperar dados dos sensores. Posteriormente, esses dados serão enviados ao servidor via internet e poderão ser acessados utilizando computadores ou Smartphones. O sistema de monitoramento de energia desenvolvido pode registrar a tensão, corrente, potência ativa, reativa, aparente, fator de potência e consumo acumulado de energia. Após a construção do protótipo, várias medidas foram efetuadas para a comprovação da viabilidade da sua utilização

Palavras-chave: Medidor de grandezas elétricas; Sistema de monitoramento de energia; Multimedidor; PZEM-004T; ESP32.

Abstract: The high cost of electricity due to generation, distribution and fees brings a single income value for several families, requiring a greater amount of information about the electricity consumption of each appliance, as well as an entire residence. Currently, there are no devices on the market for recording, recording and analyzing electrical quantities. The objective of this work is to create a prototype for the registration and monitoring of electrical quantities, intended to be implemented in the residential area. The project is based on the PZEM-004T v3 module with a capacity of up to $100 \mathrm{~A}$, using a non-invasive current sensor and an ESPDUINO32 development board to retrieve data from the sensors. Later, this data will be sent to the server via the internet and can be accessed using computers or smartphones. The developed energy monitoring system can record voltage, current, active, reactive, apparent power, power factor and accumulated energy consumption. After the prototype was built, several measures were taken to prove the viability of its use.

Key-words: Electricity meter; Energy monitoring system; Multi-meter; PZEM-004T; ESP32. 
Pode-se dizer que atualmente a energia elétrica tem um papel fundamental no planeta, podendo-se afirmar que ela é a promotora do progresso em conjunto com desenvolvimento mundial. Assim desde o seu surgimento ela propiciou a melhora no saneamento, na saúde, no abastecimento de água e alimentos, na qualidade de vida e fez surgir a sociedade capitalista e de consumo que conhecemos hoje [1].

A energia elétrica requer vários tipos de processos necessários para poder ser produzida de acordo com as necessidades, de consumo. A partir de um sistema convencional (usinas geradoras de energia, subestações e linhas de transmissão) a eletricidade é gerada, transmitida e distribuída pela concessionária local até unidades consumidoras, de modo que as perdas em cada etapa da sua distribuição tornam seu valor elevado.

Em uma escala doméstica, a energia elétrica é geralmente usada para as necessidades de equipamentos eletrônicos e eletrodomésticos, como televisores, computadores, lâmpadas, geladeira e condicionadores de ar. Cada carga possui um valor de potência diferente, quanto maior a potência, maior o custo financeiro associado à energia elétrica utilizada. $\mathrm{O}$ conhecimento da quantidade de energia gasta por cada equipamento possibilita a previsão de consumo de uma residência como um todo, possibilitando um gerenciamento do uso de cada equipamento para um menor consumo de eletricidade. Para isto, é necessário um sistema de monitoramento do consumo de energia, para que a energia elétrica possa ser usada com sabedoria.

Faz-se então necessário um gerenciamento de energia elétrica, em que os dados da medição de grandezas elétricas são um componente essencial do plano do programa de administração de energia elétrica, onde, posteriormente, os dados são usados como informações básicas na realização de auditorias e na análise do consumo de energia.

Neste trabalho, foi desenvolvido um sistema de monitoramento de grandezas elétricas com IoT (Internet das Coisas) de baixo custo que enviar os dados a uma plataforma de análise o ThingSpeak. O sistema desenvolvido pode fornecer medições detalhadas do uso de energia e dos padrões de consumo de energia. Portanto, os usuários podem entender seus padrões de uso de eletricidade.

Ao monitorar um dispositivo, um problema que costuma surgir é a distância ou o alcance que pode ser acessado pelo usuário para obter as informações necessárias e a velocidade do tempo em que as informações são obtidas. Quando a distância de monitoramento do dispositivo está apenas dentro do escopo da casa do consumidor, mídias sem fio, como Wi-Fi, Bluetooth e rádio, podem ser usadas para estabelecer comunicação. Por outro lado, se o monitoramento for necessário a uma grande distância, a Internet se mostra a melhor solução.

Devido a registradores de grandezas elétricas serem de pouca utilização no setor residencial as opções possíveis no mercado são pouco populares e custo elevado inviabilizando sua compra, o projeto proposto tenta viabilizar a construção deste instrumento utilizando material de baixo custo e fácil acesso para que possa ser montado por outros estudantes da área de eletricidade em sua residência. Assim, o projeto consiste na utilização de uma plataforma acessível e barata, o ESPDUINO32 junto com o módulo PZEM-004T V3, para a criação do medidor capaz de realizar registros de corrente, tensão e consumo acumulado de energia.

\section{REFERENCIAL TEÓRICO}

O primeiro registro de patente de um medidor de energia foi no século XIX, nos Estados Unidos, por Samuel Gardiner [2]. Basicamente, um relógio acoplado a um eletroímã. Esse medidor, operava em corrente contínua demostrando um resultado da medição na unidade de medida lâmpada-hora. A medição tinha como base o tempo em que uma lâmpada permanecia acesa.

Anos mais tarde em abril de 1888 Oliver B. Shallenberger, com base em seus experimentos na época, projetou um medidor de ampere-hora para corrente alternada, que passou a ser usado nas indústrias. Devido ao crescimento do uso da eletricidade os medidores estavam incapacitados de efetuar a leitura devido a variação de tensão e ao baixo fator de potência. A partir disto, Shallenbergerdes propôs uma melhora no seu projeto, composto por um pequeno motor de indução com as bobinas de corrente e tensão defasada em 90 graus uma da outra. Em 1903 a General Eletric (GE) lançou o primeiro medidor de corrente alternada em kWh que foi produzido em larga escala sendo usados até 1960 [3].

Atualmente existem variados modelos de medidores, distribuídos entre duas classes: o eletromecânico, que funciona a base de bobina, disco e relógio; e o eletrônico, que é composto por sensores de corrente e outro de tensão. Sua principal finalidade é medir a quantidade de energia usada por uma carga ou um conjunto delas, durante um intervalo de tempo. [4].

\subsection{Medidores Eletrônicos}


Esse tipo de medidor é atualmente mais empregado devido as várias vantagens sobre o medidor eletromecânico: tamanho menor, menor custo, sem partes móveis, mais informações sobre as grandezas elétricas além do consumo [4]. O monitoramento é feito por dois sensores, um de corrente em série com a carga que fornece uma tensão proporcional à corrente que flui através da carga, e outro para a tensão em paralelo com a carga que fornece uma tensão proporcional à tensão na carga. Os sinais provenientes desses sensores passam através de filtros anti-alising analógicos e são amostrados pelos circuitos de conversor analógico-digital (ADC). Os sinais colhidos passam por um estágio de processamento de sinal digital (DSP) a partir do qual a potência ativa é obtida. $\mathrm{O}$ valor da energia consumida pela carga é calculado integrando essa energia ao longo do tempo. A Figura 1 mostra um diagrama de blocos que exemplifica o funcionamento de um medidor eletrônico.

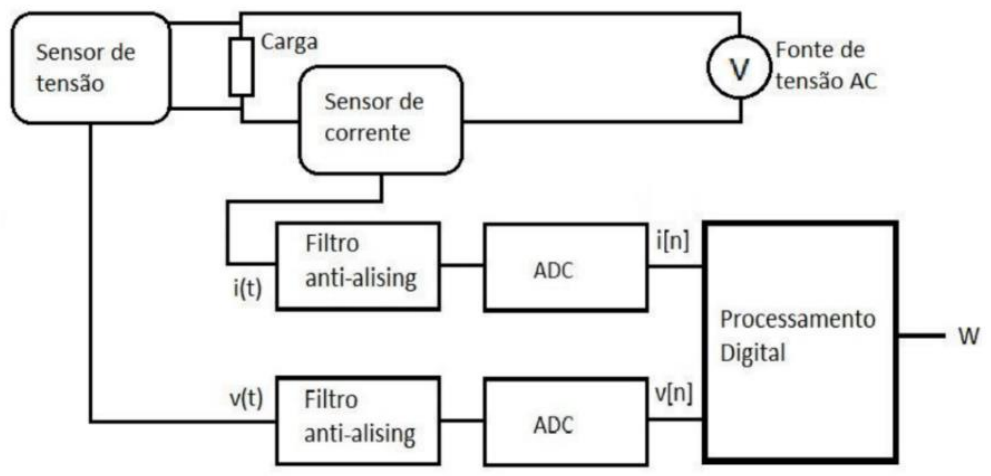

FIGURA 1. Diagrama de Bloco de um Medidor Eletrônico [4].

\subsection{Medidor Inteligente}

Um medidor inteligente é um dispositivo eletrônico que registra o consumo de energia elétrica e comunica as informações ao fornecedor de eletricidade para monitoramento e cobrança. Eles registram energia consumida e outras grandezas e os dados são enviados diariamente para os fornecedores de energia [5]. Os registradores permitem a comunicação bidirecional entre o medidor e o sistema central. Essa infraestrutura avançada de medição (AMI) difere da leitura automática do medidor (AMR), pois permite a comunicação bidirecional entre o medidor e o fornecedor. As comunicações do medidor para a rede podem ser sem fio ou por meio de conexões com fio fixas, como PLC (Power Line carrier). As opções de comunicação sem fio em uso comum incluem comunicações via rede de celulares, Wi-Fi e entre outras. Existem várias definições de medidores inteligentes e de sua constituição física, funcionalidades e meios de comunicação, onde pode-se destacar as seguintes características que definem um medidor inteligente [6]:

- Registrar em tempo real, ou próximo do real, o uso da eletricidade e a possibilidade de sua geração;

- Oferecer a possibilidade de ler as informações no local ou remotamente;

- Conectar com dispositivos pré-definidos;

- Ler outros consumos como água e gás;

- Limitar o consumo pelo medidor inteligente (em casos extremos cortar o fornecimento de energia).

\subsection{Internet das Coisas}

O termo Internet das Coisas vem do inglês Internet of Things (IoT) e significa Integração de objetos físicos e virtuais em redes conectadas à Internet, permitindo que "coisas" coletem, troquem e armazenem grandes quantidades de dados na nuvem, dos quais depois que esses dados são processados e analisados, eles geram informações e serviços em larga escala [7]. Ela possibilita a componentes físicos do dia a dia como televisores, veículos, computadores, Smartfones, sensores sejam capazes de coletar e transmitir dados ente si, se tornando assim uma extensão da internet. Essa conexão concede acesso a longa distância para controlar objetos remotamente, monitorar sensores em tempo real e usa o próprio elemento como servidor para outros aparelhos. 
O ThingSpeak é um serviço de plataforma de análise IoT, com ele pode-se visualizar, analisar fluxos e enviar comando de dados ao vivo na nuvem para dispositivos conectados. Ela fornece a visualizações instantâneas dos dados postados pelos seus dispositivos, com a capacidade de integração com o código em MATLAB $®$, Estes atrativos tornam a plataforma de fácil acesso para protótipos com sistemas de IoT sem o trabalho de criação e programação de servidores web [8]. O site disponibiliza uma licença gratuita para quem se cadastrar, porem a apresenta limites de 3 milhões mensagens por ano quatro canais diferentes e uma taxa de atualizações de mensagens de 15 segundos, retirando a instantaneidade de visualização dos dados.

\subsection{ESP32}

ESP32 é uma série microcontroladores com Wifi e Bluetooth integrados desenvolvido pela Espressif, empresa chinesa com sede em Xangai, compacto e com baixo consumo de energia sendo ideal para internet das coisas. A série ESP32 utiliza um microprocessador Tensilica Xtensa LX6 de 32-bit com processo de fabricação em 40nm, que possui clock de $160 \mathrm{MHz}$ podendo chegar até 240MHZ com duas variações uma com dois núcleos de processamento e outra com somente um. Ele integra uma antena de microfita com suporte ao padrão 802.11 b/g/n, amplificador de potência, receptor de baixo ruído amplificado, filtros, gerenciamento de energia dos módulos [9], o chip é considerado o sucessor do ESP8266.

Possui uma memória RAM $520 \mathrm{~Kb}$, suporte até 34 GPIOs sendo 8 canais PWM e com 7 entradas conversoras Analógico/Digital [10]. A tensão operacional do ESP32 varia de 2,3 V a 3,6 V. Ao usar uma fonte de alimentação única, a tensão recomendada da fonte de alimentação é de 3,3 V, sua corrente de entrada varia de acordo com as funções utilizadas por ele que fica em torno de $30 \mathrm{~mA}$ a $240 \mathrm{~mA}$ sendo a corrente da fonte aconselhada pelo fabricante de no mínimo 500mA [9].

Atualmente no mercado, existem muitas versões do ESP32 como os módulos ESP32-S, ESP32-D0WD ESP32-S0WD. Embora tenham o mesmo processador, cada um tem suas próprias características quanto a tamanho, quantidades de GPIOs, módulos integrados de câmera ou adaptador SD. A Figura 2 mostra o diagrama de pinagem do ESP-32-S.

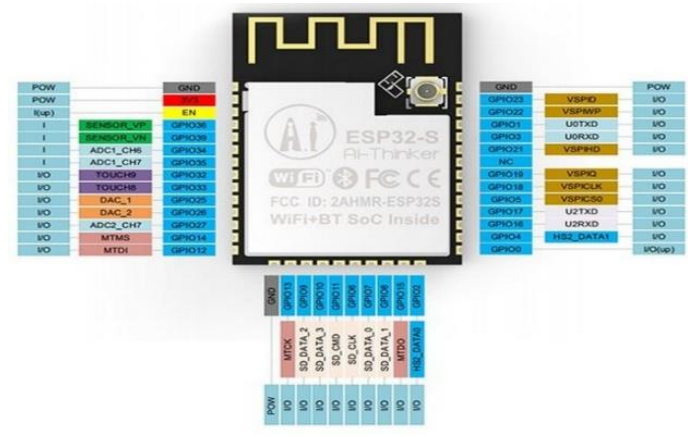

FIGURA 2. Diagrama de pinagem ESP32-S [11].

\subsection{ESPDUINO32 D1 R32}

O ESPDUINO32 D1 R32 é desenvolvido pela empresa WEMOS, que utiliza como base a placa de uma Arduino UNO R3 em conjunto com um microcontrolador ESP WROOM-32. Isto torna um produto bastante atrativo em relação aos demais, pois o mesmo dispensa o uso de uma protoboard que necessitaria ser usada no caso de uma aplicação com ESP32 sendo compatível com shields de desenvolvimento para Arduino. O chip completa a placa aliando várias funções importantes das duas plataformas com seu Wifi e Bluetooth já integrados, o regulador de tensão que permite uma tensão de alimentação entre 3,3V a 9V no pino Vin ou via conector tipo jack com uma fonte ou adaptador para bateria de $9 \mathrm{~V}$, a interface $\mathrm{CH} 340$ permite o uso de conexão USB para comunicação com o computador para transferência do código fonte do programa através uma porta micro usb [12]. A Figura 3 apresenta a placa de desenvolvimento do ESPDUINO 32 D1 R32. 


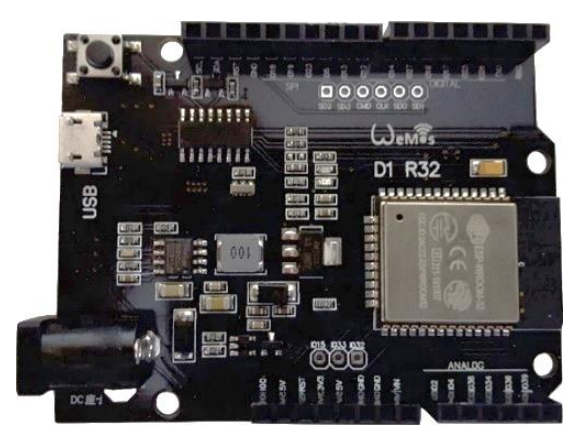

FIGURA 3. ESPDUINO32 D1 R32 [12].

A plataforma possui 18 pinos que podem ser utilizados como entradas ou saídas analógicas, onde é possível a utilização de componentes que utilizem tensões que variem entre 0 a 3,3 volts, e 28 pinos que podem ser utilizadas como entrada e saídas digitais, ou seja, pinos que operam em 0 e 3,3 volts, funcionando como chaves liga/desliga. As especificações técnicas do ESPDUINO32 podem ser vistas na Tabela 1.

TABELA 1. Especificações técnicas ESPDUINO32 D1 R32 (Adaptado de [12]).

\begin{tabular}{cc}
\hline Microcontrolador & ESP WROOM-32 \\
\hline Frequência de Clock & $240 \mathrm{MHZ}$ \\
Tensão de operação & $3,3 \mathrm{~V}$ \\
Tensão de alimentação (recomendada) & $3,3 \mathrm{~V}-9 \mathrm{~V}$ \\
Pinos digitais & 28 \\
Pinos analógicos & 18 \\
SRAM & $520 \mathrm{~KB}$ \\
Bluetooth & 4.1 \\
WiFi & $802,11 \mathrm{~b} / \mathrm{g} / \mathrm{N}$ \\
\hline
\end{tabular}

\subsection{Potência CA}

Em um circuito $\mathrm{CC}$, as tensões e correntes geralmente são constantes, que não varia com o tempo. No entanto, em um circuito CA, os valores instantâneos de tensão, corrente variam com o tempo em uma forma senoidal, deste modo a potência instantânea esta mudando constantemente, sendo influenciados pela fonte. Portanto, não podemos calcular a potência nos circuitos CA da mesma maneira que nos circuitos CC.

Assim é necessário estabelecer conceitos de potência complexa em Volt-Ampere (VA) sendo o produto dos fasores de tensão RMS com conjugado complexo de corrente RMS de acordo com a equação 1 . Por ser um número complexo, sua parte real é a potência ativa $\mathrm{P}$ em Watts $(\mathrm{W})$ e sua parte imaginária é a potência reativa Q em Volt-Ampere reativo (VAr) apresentado na Equação 2, sendo o seu módulo a potência aparente como a equação 3 demostra. Sua análise e de extrema importância por conter todas as informações pertinentes sobre potência absorvida por uma determinada carga ou conjunto delas [13].

$$
\begin{aligned}
& \mathrm{S}_{\mathrm{c}}=\mathrm{V}_{\mathrm{RMS}} \mathrm{I} *_{\mathrm{RMS}} \\
& \mathrm{S}_{\mathrm{c}}=\mathrm{P}+\mathrm{jQ} \\
& \mathrm{S}_{\mathrm{ap}}=\sqrt{\mathrm{P}^{2}+\mathrm{Q}^{2}}
\end{aligned}
$$

Já a potência ativa real P é a potência média em watts liberada para uma carga; ela é a única potência útil e real dissipada pela carga. É determinada pelo produto dos módulos dos valores de corrente e tensão eficazes e o cosseno da diferença de fase entre tensão e corrente de acordo com a Equação 4 [13]. 


$$
\mathrm{P}=\mathrm{V}_{\mathrm{RMS}} \mathrm{I}_{\mathrm{RMS}} \operatorname{Cos}\left(\theta_{\mathrm{v}}-\theta_{\mathrm{i}}\right)
$$

Essas duas grandezas se relacionam de acordo com o fator de potência que é um número entre 0 e 1 que significa qual percentual da potência aparente está sendo transformada em potência ativa constituindo um indicador de qualidade da energia, que pode ser obtido de duas formas, cosseno da diferença de fases entre tensão e corrente como demostrado na Equação 5 ou pela razão da potência ativa sobre a aparente apresentada na equação 6 [13].

$$
\begin{gathered}
\mathrm{fp}=\operatorname{Cos}\left(\theta_{\mathrm{v}}-\theta_{\mathrm{i}}\right) \\
\mathrm{fp}=\frac{\mathrm{P}}{\mathrm{S}_{\mathrm{ap}}}
\end{gathered}
$$

- Para uma carga puramente resistiva, a tensão e a corrente estão em fase, de modo que $\theta_{v}-\theta_{i}=0^{\circ}$ $\mathrm{fp}=1$;

- Para uma carga puramente reativa, a corrente esta adianta ou atrasada do tesão $\theta_{v}-\theta_{i}= \pm 90^{\circ}$ é o $\mathrm{fp}=0$;

- Para uma carga mista que pode ser capacitiva ou indutiva, a corrente esta adianta ou atrasada do tesão de modo que $0<\theta_{v}-\theta_{i}<+90^{\circ}$ ou $0>\theta_{v}-\theta_{i}>-90^{\circ}$ é o $0<f p<1$.

Agência Nacional de Energia Elétrica ANEEL através da resolução 414 determina que o fator de potência indutivo ou capacitivo não deve ser inferior a 0,92 para o grupo A com fornecimento em média tensão [14], com aplicação de multa caso houver algum consumo de potência reativa excedente ao estipulado. Para consumidores de baixa tensão do grupo $\mathrm{B}$, a norma estabelece que as concessionárias não podem cobrar excedente de reativo, realizando somente a leitura da potência ativa através um wattímetro eletromecânico ou eletrônico [14]. Portanto, para que informações precisas de consumo sejam apresentadas, é necessário medir a potência ativa ao longo do tempo. Para o cálculo de potência ativa e necessário a potência aparente multiplicado pelo fator de potência de acordo com a Equação 7, onde o fator de potência e obtido através de um medidor que consegue ler a defasagem da corrente em relação a tensão como referência [4].

\subsection{Consumo de Energia Elétrica}

A unidade base para a tarifação da energia elétrica usada pelas distribuidoras é o $\mathrm{kWh}$. O valor e dado através da utilização de medidores popularmente chamados "relógios" eletromecânicos ou eletrônicos instalados no padrão de entrada das unidades de baixa tensão. Eles utilizam a Equação (7) em que os valores medidos em pequenos intervalos de tempo são aproximados através de uma integral numérica [4]. A energia dada pela integral do produto do módulo da tensão e corrente eficazes instantâneas vezes o fator de potência em um período de tempo de acordo com a equação 7 [4].

$$
\mathrm{E}=\int_{\mathrm{t}_{0}}^{\mathrm{t}} \mathrm{V}_{\mathrm{RMS}}(\mathrm{t}) \mathrm{I}_{\mathrm{RMS}}(\mathrm{t}) \operatorname{Cos}\left(\theta_{\mathrm{v}}-\theta_{\mathrm{i}}\right) d \mathrm{t}
$$

\subsection{PZEM-004T V3}

O núcleo do módulo é constituído de um SoC V9881d, desenvolvido pela Vango Technologies. A série de chips SoC da família V98XX de e atribuído a mensuração de energia em rede monofásica, o dispositivo conta com consumo de energia muito baixo e alto desempenho. Ele integra o Analog Front-End (AFE), arquitetura de medição de energia, $8052 \mathrm{MCU}$ núcleo aprimorado, RTC, WDT, memória Flash, RAM e disponibilidade para driver de LCD [15]. Pode ser usado para aplicações de medidores de energia multifuncionais monofásicos.

O módulo PZEM-004T é um entre vários modelos de medidores de grandezas elétricas produzidos pela chinesa Peacefair. Sendo a terceira versão utilizada neste trabalho, trazendo recursos extras como dados de fator de potência e frequência da rede. $\mathrm{O}$ módulo deve ser alimentado com um nível de tensão de $5 \mathrm{~V}$ e tem mesmo valor logico de saída comunicação serial TTL. Estas propriedades tornam uma ótima escolha projeto DIY, onde precisa-se de informações sobre o consumo de energia sendo compatível com Arduino / ESP32 / Raspberry Pi. 
Entre seus vários dados temos as medidas de corrente e tensão em valor eficaz, cálculo interno de potência ativa e uma memória que armazena desde os primeiros valores da energia acumulada para caso o módulo perda sua alimentação, a memória pode ser resetada através de um comando. A Figura 4 apresenta o módulo medidor PZEM.

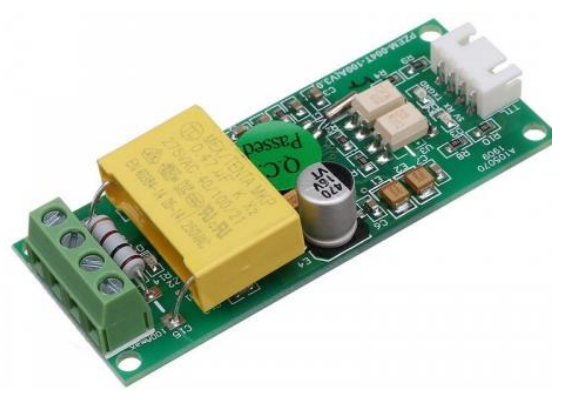

FIGURA 4. PZEM-004T V3 [16].

Há um faixa de medição que são um conjunto de valores mensurando que admitimos um erro do instrumento de medição é mantido dentro do limite, ela pode ser menor ou, no máximo, igual a faixa nominal. Os valores da faixa de medição e de precisão de cada grandeza segundo a Peacefair podem ser vistos na Tabela 2.

TABELA 2. Características elétricas PZEM-004T V3 (Adaptado de [17]).

\begin{tabular}{ccc}
\hline Grandezas & Faixa de medição & Precisão \\
\hline Tensão & $80-260 \mathrm{~V}$ & $0,5 \%$ \\
Corrente & $0-100 \mathrm{~A}$ & $0,5 \%$ \\
Potência ativa & $0-23 \mathrm{~kW}$ & $0,5 \%$ \\
Fator de potência & $0,00-1,00$ & $1,0 \%$ \\
Frequência & $45-65 \mathrm{~Hz}$ & $0,5 \%$ \\
Energia & $0-9999,99 \mathrm{kWh}$ & $0,5 \%$ \\
\hline
\end{tabular}

\subsection{Sensor de Corrente}

Os transformadores de corrente TC, também chamados de transformadores de instrumentos, são utilizados em aplicações de alta corrente e têm a função de fornecer correntes proporcionais às medidas no circuito primário de potência, mas suficientemente reduzidas e isoladas galvanicamente de forma a possibilitar a medição por equipamentos de proteção e medição [4]. O modelo do PZEM escolhido acompanha um instrumento de medição TC tipo alicate com capacidade de até 100A de corrente, facilitando a passagem do fio pelo seu interior e segurança na instalação por não tocar diretamente nas partes vivas.

\section{MATERIAIS E MÉTODOS}

Observando que no mercado não há disponibilidade de instrumentos baratos para a realizações das medições e registros precisos de grandezas elétricas, fez-se necessário a criação de um protótipo economicamente viável constituído de um o projeto simples que pudesse ser executado com poucos componentes, Para tanto, foi decidido utilização de um módulo de medição PZEM em conjunto com um ESPDUINO32 para envio das informações a um servidor que irá armazenar os dados. A utilização da nuvem traz simplicidade ao projeto gerando economia nos custos, porém exige acesso internet via rede wifi ou por roteamento de rede móvel $3 \mathrm{G}$ via Smartfone devendo não se localizar muito distante do projeto. O problema é minimizado pelo fato da popularidade da interne sem fio que abrange grande partes das residências e ambientes públicos atualmente.

\subsection{Definição do Circuito}


As principais partes que compõem os circuitos são ESPUDINO32, e o módulo PZEM-004T V3. O monitoramento exige duas referências de dados uma de tensão obtido através de uma extensão com plug macho de 2 pinos ligada diretamente na tomada e outra de corrente obtida por meio de medição indireta com TC diminuído os ricos de choque e agilizando a montagem. O módulo depende da alimentação da tensão de referência, caso não esteja conectado a tomada com uma tensão acima de $80 \mathrm{~V}$ o mesmo não apresenta nenhum dado pela saída serial.

A placa de desenvolvimento escolhida foi o ESPDUINO32, que apesar das suas limitações com tensão de operação de $3,3 \mathrm{~V}$ se comportou normalmente com a saída serial de $5 \mathrm{~V}$ do módulo, há 4 conectores no módulo $\mathrm{Rx}, \mathrm{Tx}, 5 \mathrm{~V}$ e Gnd que serão conectados a placa nos terminais IO17, IO16, 5V e Gnd respectivamente ligados por cabos macho fêmea. $\mathrm{O}$ dispositivo foi uma escolha ideal para o trabalho e apresentou bons resultado por ter um preço acessível em relação aos outros microcontroladores, dispensou o uso de conversores DC - DC podendo se ligado diretamente a uma fonte de $9 \mathrm{~V}$ pelo jack da placa facilitando a alimentação do conjunto. Por fim o mais importante, apresentar wifi integrado para envio de dados ao ThingSpeak. A Figura 5 demostra a forma como o circuito foi montado.

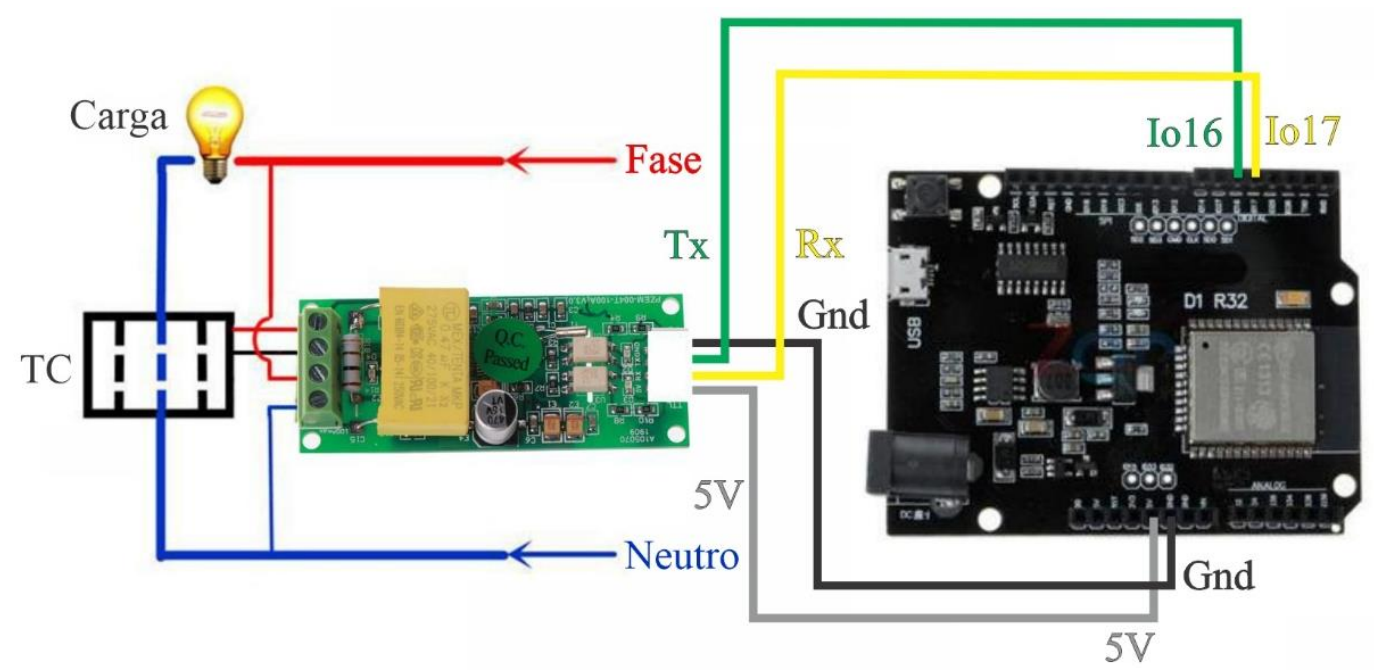

FIGURA 5. Esquema elétrico do sistema de medição de corrente e tensão (Autoria própria).

\subsection{Montagem do protótipo}

Para armazenar os elementos do projeto, buscou-se um material pré-fabricado. A caixa de passagem 4x4 foi a opção escolhida pois é facilmente encontrada em lojas de matérias de construção sendo feita de material PVC junto com uma placa cega $4 \times 4$ para fechar o conjunto. Foram feitos furos na parte inferior da caixa de passagem para serem colocados parafuso rosca total cabeça de fenda com 1/4", prendendo os elementos através de seus furos nas placas com roscas, outros furos foram feitos nas partes destacadas da caixa para passagem da extensão da tomada junto com os dois fios do TC e outro furo para passagem do cabo micro USB com conector da fonte de alimentação de $9 \mathrm{~V}$.

O protótipo necessita de duas tomadas, uma para a referência de tensão e outra para uma fonte de alimentação da placa de desenvolvimento, pode-se usar somente a extensão do módulo fazendo uma derivação para a fonte, que não foi feita neste projeto. A Figura 6 mostra o modelo final montado.

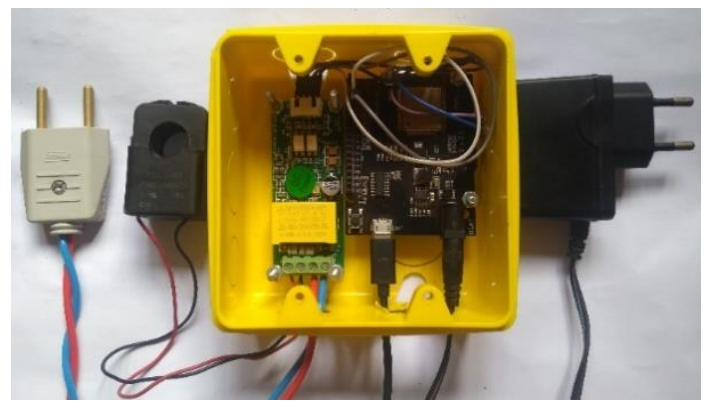


FIGURA 6: Montagem final do protótipo do sistema de monitoramento de eletricidade (Autoria própria).

\subsection{Programação}

Para desenvolvimento do código utilizou-se a IDE do Arduino, em que a plataforma na nuvem não necessitou de programação, somente configuração de campos de informações. O sistema de armazenamento e transmissão de dados escolhido foi a nuvem com a plataforma ThingSpeak necessitando de envio de informações via o ESPDUINO32. O monitoramento da rede foi realizado através do site da plataforma podendo ser observados por Smartfone ou computador através do link [18]. O PZEM obtém as informações da rede elétrica por seus sensores e enviar para o ESP32, que são mandados para nuvem via wifi para oito campo diferentes cada um responsável por armazenar e exibir os valores que são enviados a cada 15 segundos. Os oito campos criados são Tensão (V), Corrente (A), Potência Ativa (W), Consumo (Wh), Fator de potência, Frequência (Hz), Potência Aparente (VA), Potência Reativa (VAr). Como o PZEM não demostra os dados de potência aparente ou reativa, os mesmos foram das equações (6) e (3) respetivamente dentro da programação. Dentro do código a medida de $\mathrm{kWh}$ foi multiplicada por mil para ter uma maior quantidade algarismos significativos pois o aparelho somente exibia 2.

\section{RESUltAdOS}

\subsection{Custo Total do Circuito}

A Tabela 3 resume os dois principais itens do projeto com seus valores e fornecedores, em que a cotação do dólar no período da aquisição dos produtos sendo de $\mathrm{R} \$ 3,88$. Os produtos foram comprados em lojas virtuais sendo possível o acesso de qualquer um aos elementos, em que foi priorizado o baixo valor, apesar do tempo de espera para sua chegada por ser um fornecedor internacional.

TABela 3. Custo Total do Protótipo (Autoria própria).

\begin{tabular}{ccccc}
\hline Componentes & Valor unitário & Custo de Envio & Total & Fornecedor \\
\hline ESPDUINO32 & $\mathrm{R} \$ 17,56$ & $\mathrm{R} \$ 16,55$ & $\mathrm{R} \$ 34,11$ & Aliexpress.com \\
PZEM-004T V3 & $\mathrm{R} \$ 45,83$ & $\mathrm{R} \$ 9,51$ & $\mathrm{R} \$ 55,34$ & Aliexpress.com \\
& TOTAL & & $\mathrm{R} \$ 89,45$ & \\
\hline
\end{tabular}

O custo do conjunto pode ser reduzido utilizando um ESP8266 com preço mais acessível e um de PZEM com TC do tipo fixo, porém deixando o produto com uma dificuldade maior de instalação. Uma pesquisa no mercado chinês mostrou produtos similares mais barato como o Din Rail LED Voltmeter Ammeter da LEDSMITH por USD\$14,90 e um outro AT3010-LY da HIDANCE com aplicativo para comunicação com Smartfone via Blutooth por USD\$11,08. Todo o produto tem funções parecidas tensão, corrente, potência ativa, consumo acumulado de energia, fator de potência, sendo seus preços citados sem o custo de envio. O projeto do artigo se mostra mais caro, mas com uma vantagem em relação aos outros, um registro das grandezas elétrica na nuvem para uma posterior análise e visualização dos dados a longa distâncias.

\subsection{Testes}

Foram feitos vários testes para comprovar a precisão do módulo com vários tipos de cargas. O local de teste foi o laboratório máquinas elétricas da UFERSA, com bancadas de simulação de cargas colocadas em série e conectadas a tensão da rede em $220 \mathrm{~V}$. As leituras do protótipo com cargas foram feitas simultaneamente com um alicate wattímetro digital modelo ET-4090 da Minipa, para comparação de valores. As cargas são resistores de $190 \Omega$, indutores de $363 \mathrm{mH}$ e capacitores $19 \mathrm{uF}$. Não foram simuladas cargas puramente indutivas ou capacitivas, devido a ser incomum em uma residência cargas desse tipo. A Equação 8 mostra como foi calculado o erro percentual.

$$
\operatorname{Erro}(\%)=\left|\frac{\text { Grandeza wattímetro }- \text { Grandeza pzem }}{\text { Grandeza wattímetro }}\right| \times 100 \%
$$


O Quadro 1 demostra os valores dos testes obtidos com o PZEM em comparação com o wattímetro digital da Minipa sendo estes nossa referência de para a tomado do erro de cada medição.

QUADRO 1. Comparação de valores (Autoria própria).

\begin{tabular}{|c|c|c|c|c|c|c|}
\hline Carga & $\begin{array}{l}\text { Instrumento } \\
\text { de medição }\end{array}$ & Tensão (V) & $\begin{array}{c}\text { Corrente } \\
\text { (A) }\end{array}$ & $\begin{array}{l}\text { Potência } \\
\qquad(W)\end{array}$ & $F P$ & $\begin{array}{l}\text { Potência } \\
\qquad(V A)\end{array}$ \\
\hline \multirow{3}{*}{1 Resistor } & Wattímetro & 229,6 & 1,6 & 367,9 & 0,99 & 365,7 \\
\hline & PZEM & 229,7 & 1,65 & 379,1 & 1 & 379,1 \\
\hline & $\operatorname{Erro}(\%)$ & $0,04 \%$ & $3,12 \%$ & $3,04 \%$ & $1,01 \%$ & $3,66 \%$ \\
\hline \multirow{3}{*}{4 Resistores } & Wattímetro & 230,6 & 0,42 & 97,8 & 0,99 & 94,5 \\
\hline & PZEM & 230,1 & 0,41 & 94,8 & 1 & 94,5 \\
\hline & Erro(\%) & $0,22 \%$ & $2,38 \%$ & $3,07 \%$ & $1,01 \%$ & $0,00 \%$ \\
\hline \multirow{3}{*}{9 Resistores } & Wattímetro & 230,4 & 0,2 & 46,6 & 0,99 & 46,1 \\
\hline & PZEM & 229,7 & 0,18 & 41,6 & 1 & 41,8 \\
\hline & $\operatorname{Erro}(\%)$ & $0,30 \%$ & $10,00 \%$ & $10,73 \%$ & $1,01 \%$ & $9,33 \%$ \\
\hline \multirow{3}{*}{$\begin{aligned} & 1 \text { Resistor } \\
+ & 1 \text { Capacitor }\end{aligned}$} & Wattímetro & 231,4 & 1,2 & 213,8 & 0,73 & 278,4 \\
\hline & PZEM & 230,6 & 1,2 & 192,5 & 0,7 & 275,7 \\
\hline & $\operatorname{Erro}(\%)$ & $0,35 \%$ & $0,00 \%$ & $9,96 \%$ & $4,11 \%$ & $0,97 \%$ \\
\hline \multirow{3}{*}{$\begin{array}{c}1 \text { Resistor } \\
+2 \text { Capacitores }\end{array}$} & Wattímetro & 235,7 & 0,77 & 90,4 & 0,48 & 186,1 \\
\hline & PZEM & 232,4 & 0,77 & 76,8 & 0,3 & 178,84 \\
\hline & $\operatorname{Erro}(\%)$ & $1,40 \%$ & $0,00 \%$ & $15,04 \%$ & $37,50 \%$ & $3,90 \%$ \\
\hline \multirow{3}{*}{$\begin{array}{c}1 \text { Resistor } \\
+3 \text { Capacitores }\end{array}$} & Wattímetro & 234,4 & 0,56 & 48,3 & 0,36 & 131,7 \\
\hline & PZEM & 232,2 & 0,55 & 36,8 & 0,29 & 126,9 \\
\hline & Erro(\%) & $0,94 \%$ & $1,79 \%$ & $23,81 \%$ & $19,44 \%$ & $3,64 \%$ \\
\hline \multirow{3}{*}{$\begin{array}{l}1 \text { Resistor } \\
+1 \text { Indutor }\end{array}$} & Wattímetro & 229,5 & 1,07 & 170,6 & 0,79 & 246,9 \\
\hline & PZEM & 228,5 & 1,03 & 167,7 & 0,7 & 239 \\
\hline & $\operatorname{Erro}(\%)$ & $0,44 \%$ & $3,74 \%$ & $1,70 \%$ & $11,39 \%$ & $3,20 \%$ \\
\hline \multirow{3}{*}{$\begin{aligned} & 1 \text { Resistor } \\
+ & 2 \text { Indutores }\end{aligned}$} & Wattímetro & 231,5 & 0,57 & 72,1 & 0,55 & 131,1 \\
\hline & PZEM & 229,8 & 0,6 & 64 & 0,47 & 135,96 \\
\hline & $\operatorname{Erro}(\%)$ & $0,73 \%$ & $5,26 \%$ & $11,23 \%$ & $14,55 \%$ & $3,71 \%$ \\
\hline \multirow{3}{*}{$\begin{aligned} & 1 \text { Resistor } \\
+ & 3 \text { Indutores }\end{aligned}$} & Wattímetro & 230,6 & 0,39 & 28,13 & 0,3 & 90,4 \\
\hline & PZEM & 227,6 & 0,4 & 35,16 & 0,37 & 91,9 \\
\hline & $\operatorname{Erro}(\%)$ & $1,30 \%$ & $2,56 \%$ & $24,99 \%$ & $23,33 \%$ & $1,66 \%$ \\
\hline
\end{tabular}

Com os testes foi possível observar uma variação percentual entre a leitura do módulo em relação a wattímetro da Minipa. As cargas resistivas obtiveram baixa variação entre os dois dispositivos de medição, devido ao fator de potência unitário. As cargas capacitivas e indutivas apresentaram uma variação especialmente nas medidas de potência ativa quando o valor do fator de potência é reduzido, o que pode ser explicado pela Equação 4 em que a potência ativa é diretamente proporcional ao valor do fator de potência.

\subsection{Aplicação}

O protótipo foi ligado diretamente ao padrão de entrada de uma residência, durante o período de uma semana, os testes iniciaram em quinze de janeiro às doze horas e foram até o dia vinte e dois de janeiro no mesmo horário. O ThingSpeak armazenou os dados enviados pelo ESPUINO32 em uma planilha eletrônica que 
foi baixada e feita uma análise dos dados coletados para criação de gráficos. A Figura 7 apresenta o histórico de potência demandada pela residência em um período de um dia.

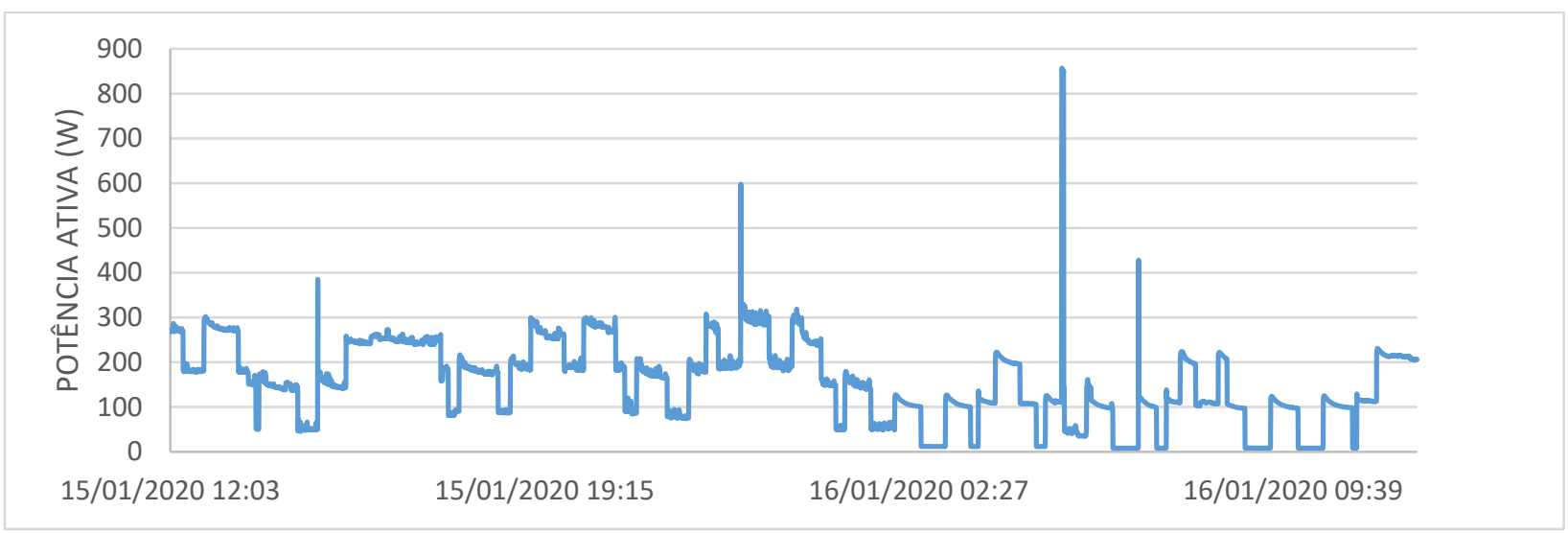

FIGURA 7. Leitura de potência ativa PZEM-04T V3 ThingSpeak (Autoria própria).

A leitura de energia demostrou que foram consumidos neste período $23,90 \mathrm{kWh}$, para fins de análise de em um mês considerou-se que as três semanas seguintes teriam um gasto semelhante. Obtendo assim uma previsão de consumo para o mês de análise de $95,6 \mathrm{kWh}$, que se aproximou do valor da fatura mais recente do local de $102 \mathrm{kWh}$. Por não se ter realizada a análise comparativa do consumo está leitura foi considera apenas uma estimativa. A Figura 8 abaixo apresenta o histórico de consumo de energia a cada dia.

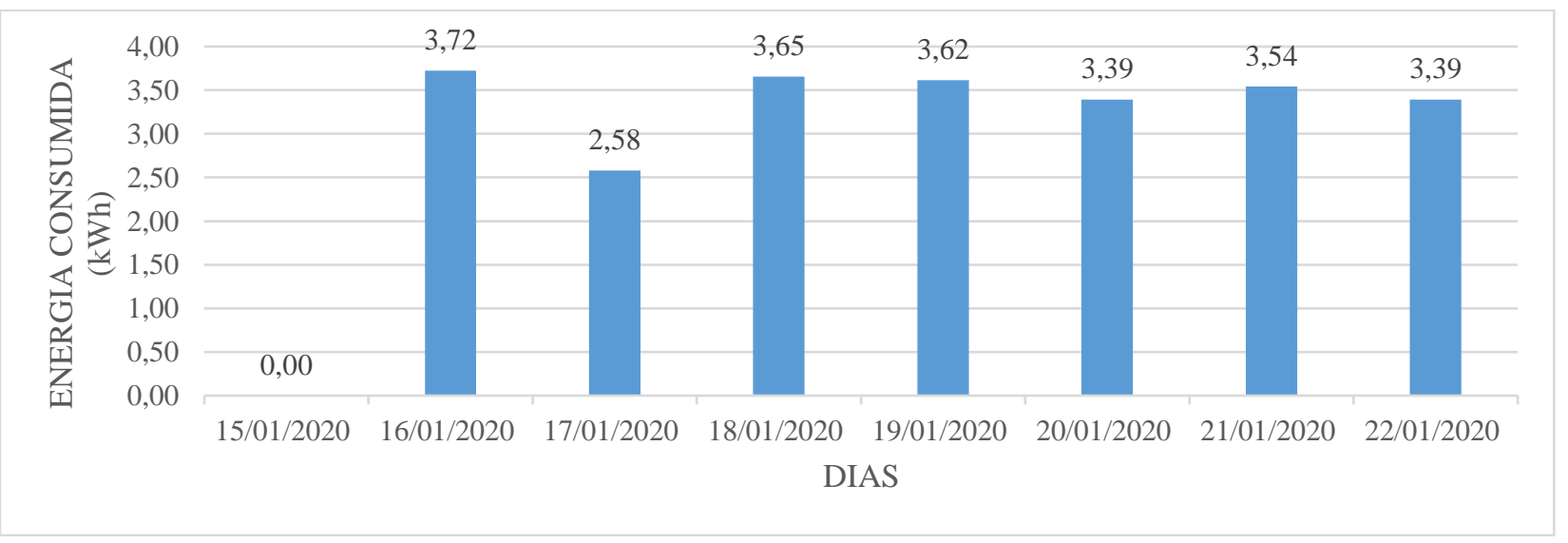

FIGURA 8. Leitura de consumo de energia PZEM-04T V3 ThingSpeak (Autoria própria).

\section{CONCLUSÃO}

O trabalho obteve êxito em criar um protótipo de medidor de grandezas elétricas com acesso remoto de baixo custo para a área residencial, não foi possível verificar a precisão de leitura do consumo de energia devido a não disponibilidade de um registrador de grandezas elétricas ou analisador de qualidade de energia na data dos testes. Foi obtido sucesso nos objetivos propostos, tendo um preço próximo em relação a outros medidores já citados das marcas LEDSMITH e HIDANCE, que são invasivos, mas o projeto proposto conta com a função extra de armazenar as informações em uma plataforma de IoT e possuir visualização a distância.

Existe maneiras de reproduzir o projeto com custos reduzidos utilizando a prototipagem e comprando somente os componentes eletrônicos necessários para recriar o circuito, podendo ser reproduzido em larga escala. O protótipo pode ser melhorado em alguns aspectos, tais como um display para visualização dos dados em tempo real, adaptação dos códigos para a envio de informações via Bluetooth para um Smartfone sem acréscimo nenhum de custo. Outro ponto de melhoria é o armazenamento de informações na nuvem dependente da rede wifi, tal problema poderia ser contornado utilizando a nuvem em conjunto com módulos SD e RTC para salvar informações em uma mídia física com data e hora em caso de perda da conexão com a internet.

O ThingSpeak se comportou como uma ótima plataforma na sua versão gratuita diminuindo consideravelmente o tempo da criação do projeto pelo fato de não necessitar de programação, ele ainda possui 
uma boa compatibilidade com Smartfone em que o sistema de exportação de dados via planilha no formato csv facilita a análise das informações, a ressalva do uso da ferramenta e o delay imposto pelo site de 15 segundos para o envio de informações retirando a visualização em tempo real das medidas do projeto. Uma sugestão para trabalhos futuros seria uma pesquisa de outras plataformas que tivessem os mesmos pontos positivos da primeira junto com instantaneidade de envio de informações para os usuários.

\section{REFERÊNCIAS}

[1] GOMES, J. P. P.; VIEIRA, M. M. F. O campo da energia elétrica no Brasil de 1880 a 2002. Rev. Adm. Pública, Rio de Janeiro, v. 43, n. 2, p. 295-321, Abril, 2009.

[2] LIMA, A. V.; PEREIRA, V. O. Transmissão Automática de Dados de Energia TAD-E. 2011, Espírito Santo, Brasil. Monografia Graduação - Departamento de Engenharia Elétrica, Faculdade Novo Milênio.

[3] VAZ, L. M. M. Impactos e Benefícios do Sistema de Medição Centralizada. Estudo de caso numa concessionária brasileira de eletricidade. Rio de Janeiro, 2012. Dissertação de Mestrado - Departamento Pós-MQI, Pontifícia Universidade Católica do Rio de Janeiro.

[4] MORO, J. Z. Medidor de Consumo de Energia Elétrica Descentralizado e com Interface WEB. 2013. 80 f. Dissertação (Mestrado) - Curso de Engenharia Elétrica, Faculdade de Engenharia Elétrica e de Computação, Universidade Estadual de Campinas, Campinas, 2013.

[5] CESÁRIO JÚNIOR, J. M. Um medidor de Energia Elétrica Integrado em Redes de Comunicação. 2014. 95 f. Dissertação (Mestrado) - Curso de Tecnologia, Faculdade de Tecnologia, Universidade Estadual de Campinas, Limeira, 2014.

[6] FEDERAL ENERGY REGULATORY COMMISSION. Assessment of Demand Response and Advanced Metering. Washington: 2008.

[7] INTERNET DAS COISAS: Nós, as cidades, os robôs, os carros: tudo conectado!. [s.i.]: Sociedade Brasileira de Computação, abr. 2015.

[8] THINGSPEAK. Saiba mais sobre ThingSpeak. Disponível online:< https://thingspeak.com/pages/learn_more> (acesso em 19/01/2020).

[9] SYSTEMS, Espressif. ESP32EX: Datasheet. 3.3. ed. Espressif Systems. 2019.

[10] KOLBAN, Neil. Kolban's book on ESP32. [S.1.]: Leanpub, 2018.

[11] 99 TECH. ESP32-S WiFi \& Bluetooth Module with ESP32 and PCB/IPEX(u.fl) Antenna (2019). Disponível online: <https://99tech.com.au/product/esp32-s-ai/> (acesso em 18/01/2020).

[12] STEFAN'S WEBBLOG. WeMos D1 R32 ESP32 (2019). Disponível em:<http://fambach.net/wemos-d1r32-esp32/ > (acesso em 18/01/ 2020).

[13] ALEXANDRE, C. K.; SADIKU, M. N. O. Fundamentos de Circuitos Elétricos. 5. ed. Porto Alegre: AMGH, 2013.

[14] BRASÍLIA. Resolução normativa ANEEL. n 414/2010 de 09 de setembro de 2010. Disponível online: <http://www.aneel.gov.br/cedoc/ren2010414.pdf> (acesso em 18/01/2020).

[15] Technologies, Vango. V98XX Datasheet. 3 ed. Vango Technologies. 2018.

[16] INNOVATORS GURU. PZEM-004T V3 (2019). Disponível online: <https://www.innovatorsguru.com/pzem-004t-v3/> (acesso em 19/01/2020).

[17] ELECTRONIC TECHNOLOGY, Peacefair. PZEM-004T V3 Datasheet user manual. 1. Ed. Peacefair Electronic Technology. 2019.

[18] THINGSPEAK. Medidor de Grandezas Elétricas. Disponível online:<https://thingspeak.com/channels/928879> (acesso em 19/01/2020). 